\title{
Application of Improved Template Matching Algorithm inTarget Image Detection
}

\author{
Guoquan Ren ${ }^{1}$, a Wentao Jiang ${ }^{1}$, Dongwei $\mathrm{Li}^{1}$, Guozhang $\mathrm{Li}^{1}$ \\ ${ }^{1}$ Ordnance Engineering College, Shijiazhuang, 050003, China \\ aemail:709955574@qq.com
}

\begin{abstract}
Keywords: Target Detection, Template Matching, Normalized Correlation, Optimal Searching Step-time
\end{abstract}

\begin{abstract}
In view of the disadvantage of the current method for target detection that poor efficiency and poor real-time, a novel method of target detection based on digital image processing technology was proposed. The traditional normalized correlation template matching algorithm was improved via setting optimal searching step-time in that method, which can greatly reduce the amount of computation and enhance the matching speed. The experimental results show that the proposed algorithm has high accuracy and high speed, and it can be applied in target image detection effectively.
\end{abstract}

\section{Introduction}

With the rapid development of modern weapon system, target range test technology level has been improved ${ }^{[1,2]}$. At present, template matching method is widely employed in the target detection system. Normalized correlation measurement matching algorithm(NC) is one of the most used template matching algorithm, because of its high accuracy and adaptability ${ }^{[3]}$. However, this method usually has large amount of calculation and slow speed and so on by adopting exhaustive search way, which affects it in the actual application. In this paper, starting from the aspect of reducing matching frequency of search point, a optimal searching step-time $\mathrm{NC}$ algorithm is proposed by improving the traditional NC algorithm, which improves the matching speed on the basis of the matching precision.

\section{Target image detection system}

In the target detection experiment, the method is to install device for obtaining line of sight in the rear of the eyepiece of artillery sighting telescope to absorb the sight view the central part of the image $^{[4]}$. As is shown in Fig. 1, the experiment system consists of adjustable beam splitter, steering prism, high-speed CCD, high-speed image acquisition card and computer image processing.
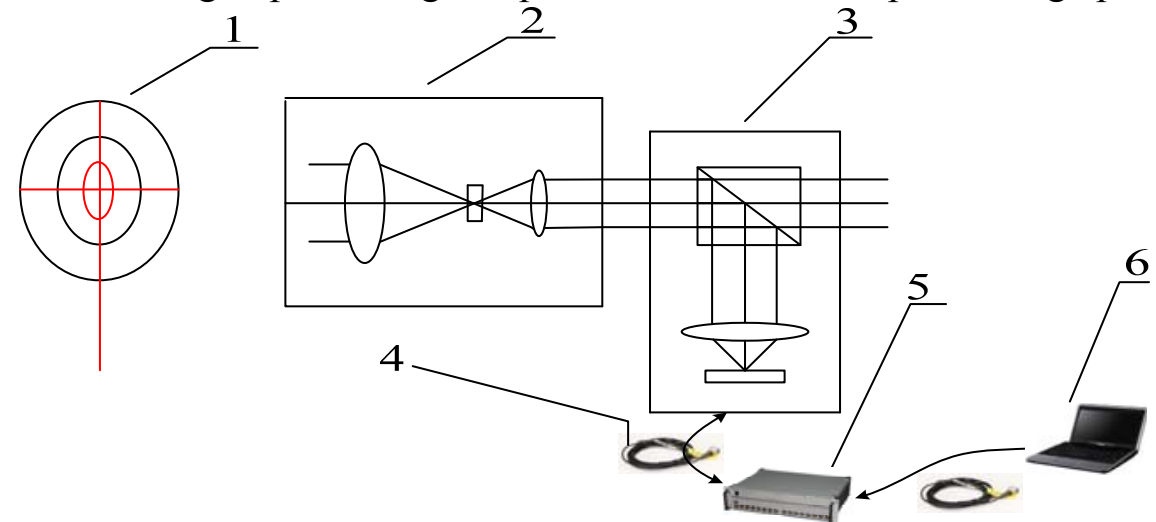

1 - target; 2 - artillery sighting telescope; 3 -high-speed CCD;

4 - video transmission line; 5 - high-speed image acquisition card; 6 - computer image processing

Fig.1 Composing and setting of experiment system

In the process of experiment, Its working principle is to separate the light from artillery sighting telescope eyepiece according to a certain proportion. After turning the image by prism, the camera 
lens focus image on CCD target surface, and then image on CCD surface of the target will be collected by a high-speed image acquisition card. At the same time, through the data bus the data is transmitted to the computer. Finally, target location is determined through template matching method.

\section{Improved template matching algorithm}

Template matching ${ }^{[5,6]}$ refers to determine whether there is the target which is the same as size and direction of template image in the reference images, then find the template image and confirm its coordinate position through a certain algorithm. The method search the matching image in another image according to the known template image.

\subsection{Traditional NC algorithm}

The basic idea of the $\mathrm{NC}$ algorithm ${ }^{[7]}$ is to determine the degree of match between the reference image and the template image by calculating cross-correlation measurement value. The size of the cross-correlation value between the template image and the reference image represents similarity. The greater the measurements value, the higher the degree of similarity.

As is shown in Fig.2, suppose the template image $T(X \times Y)$ moves on the reference image $S(M \times N)$. The area under the cover of template image is called search sub-image $S^{i, j}$. Where $i, j$ called reference point of search sub-image are the coordinates that the upper left pixels of the sub-image in the image $S(M \times N)$.

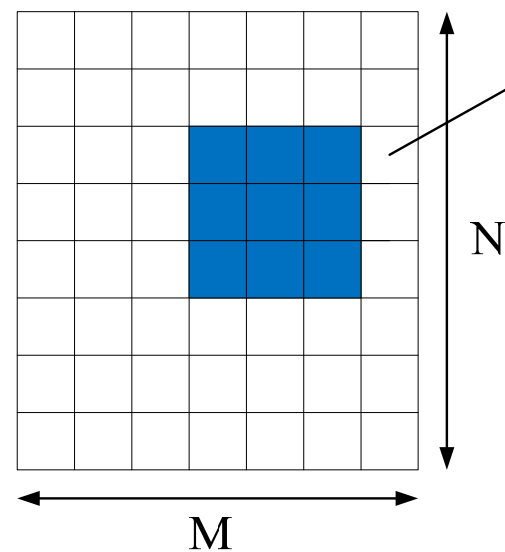

(a) reference image $S(M \times N)$ search sub-image

$\mathrm{N}$

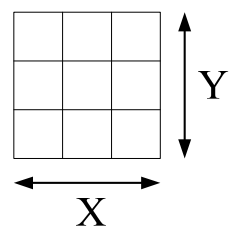

(b) template image $T(X \times Y)$

Fig. 2 Template matching process

Type (1) method is used to measure similarity degree between the template image $T(X \times Y)$ and the search sub-image $S^{i, j}$.

$$
R(i, j)=\frac{\sum_{m=1}^{M} \sum_{n=1}^{N}\left[S^{i, j}(m, n) \times T(m, n)\right]}{\sqrt{\sum_{m=1}^{M} \sum_{n=1}^{N}\left[S^{i, j}(m, n)\right]^{2}} \times \sqrt{\sum_{m=1}^{M} \sum_{n=1}^{N}[T(m, n)]^{2}}}
$$

Where, $S^{i, j}(m, n)$ is the grey value of $(m, n)$ in the search sub-image $S^{i, j}$, and $T(m, n)$ is the grey value of $(m, n)$ in the template image $T(X \times Y)$. If $R(i, j)$ of the Type (1) is equal to 1 , the template image and the search sub-image match exactly.

Traditional NC algorithm need to calculate all pixels in the reference image, while best match point is only one, all the rest of the calculation are invalid. Especially when the template image and the reference image are larger, the amount of calculation is large, which leads to a slow speed and a long calculating time, and sometimes traditional $\mathrm{NC}$ algorithm can't meet the real-time demand. Therefore, under the premise that ensures the matching quality, there is necessary to increase the matching speed by improving and optimizing the algorithm. 


\subsection{Improved NC algorithm}

On the basis of the template image and the reference image are determined, in order to enhance operation speed, it must be effective to reduce the search position. Some scholars ${ }^{[8]}$ improved the traditional NC algorithm, and the fixed step NC algorithm was proposed, but its speed and accuracy relate to the step length. When step is too small, speed is not improved obviously. When step is too big, it is easy to lose the best matching position. On this basis, the template matching is divided into coarse positioning and fine positioning based on the idea of "first rough then precise" in this paper, and the optimal searching step-time NC algorithm is proposed.

In order to guarantee the accuracy of the algorithm, this paper puts forward the following three principles:

1) Step is not allowed to exceed the size of template image, otherwise the template image can't achieve continuously translation in the reference image, which leads to decline in matching accuracy.

2) In the process of rough localization, the template image is not allowed to translate the boundary of the reference image.

3) In the process of precise localization, the template image is not allowed to translate the boundary of the reference image.

The specific steps of the algorithm are as follows:

1) Seeking optimal searching step-time. The template image both has lateral translation and vertical translation in the reference image, so the optimal searching step-time is divided into vertical step and horizontal step. Suppose the size of template image is $(X \times Y)$, the size of reference image is $(M \times N)$, horizontal step is $u(1 \leq u \leq X)$, vertical step is $v(1 \leq v \leq Y)$, it can be derived:

The translation number $C 1$ of template image in the process of rough localization is:

$$
C 1=((M-X) / u) \times((N-Y) / v)
$$

The translation number $C 2$ of template image in the process of precise localization is:

$$
C 2=(2 * u+1) \times(2 * v+1)
$$

The translation number $C$ of template image in the whole matching process is:

$$
C=C 1+C 2=((M-X) / u) \times((N-Y) / v)+(2 * u+1) \times(2 * v+1)
$$

The less the translation number of template image in the reference image, the faster the operation speed. It can find out the optimal horizontal step $u$ and vertical step $v$ by using cross validation method.

2) The process of rough localization. As is shown in Fig.3, setting the optimal horizontal step $u$ and vertical step $v$, reference point of search sub-image is translated according to the set step. The template image implements global search roughly in the reference image, and it can get the highest rough matching point.

3) The process of precise localization. As is shown in Fig.4, With rough matching point as the center, the pixel of the optimal horizontal step $u$ is extended horizontally symmetrically in horizontal direction, and the pixel of the optimal vertical step $v$ is extended vertically symmetrically in vertical direction. Reference point of search sub-image is translated gradually in transverse and longitudinal direction within the extensional scope, and the template image implements partial search precisely in the reference image, and it can get the highest precise matching point. 


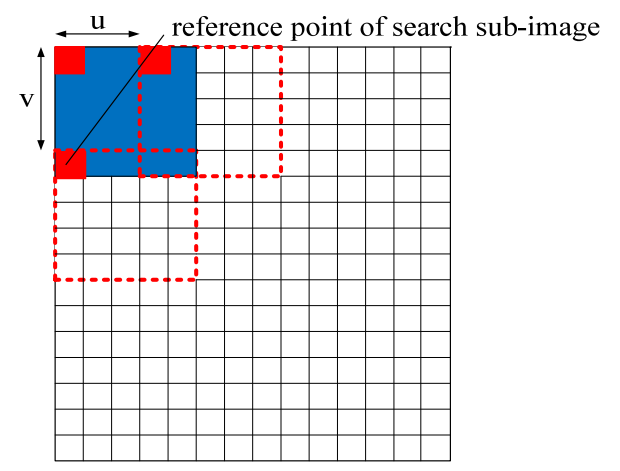

Fig. 3 The process of rough localization

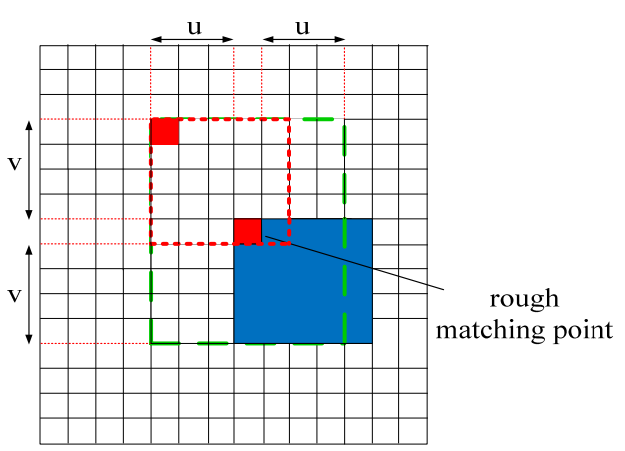

Fig.4 The process of precise localization

\section{Experimental result and analysis}

The optimal searching step-time NC algorithm is achieved on the computer, and the sequence images absorbed with CCD are detected, which obtain the good effect. In the process of target detection, as is shown in Fig.5 and Fig.6, the size of reference image is $360 \times 280$, and the size of template image is $90 \times 80$.

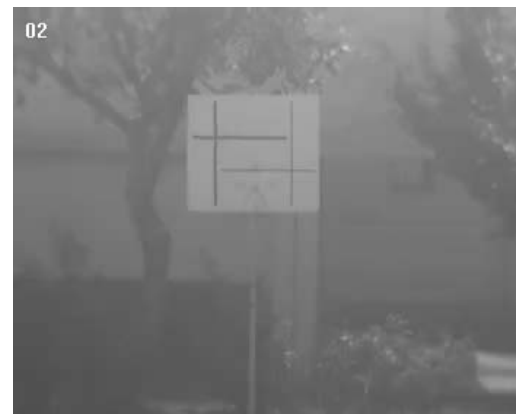

Fig.5 Reference image

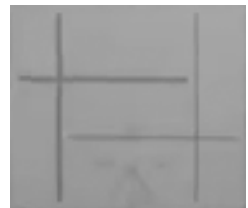

Fig.6 Template image

\subsection{The comparison of accuracy}

The target image is detected by using the traditional NC algorithm, the fixed step NC algorithm and the optimal searching step-time NC algorithm respectively. The matching results are as shown in Fig.7 and Tab.1.

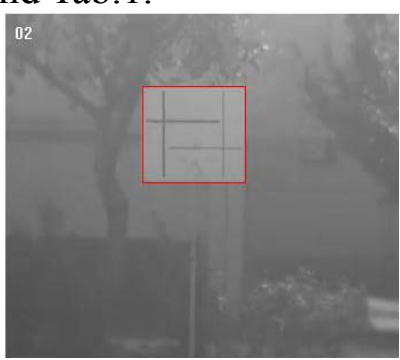

(a) traditional $\mathrm{NC}$ algorithm(TRD)

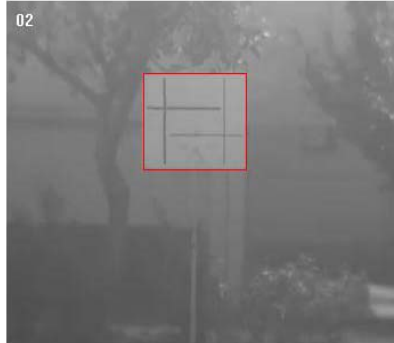

(b) fixed step $\mathrm{NC}$ algorithm(FIX(1))

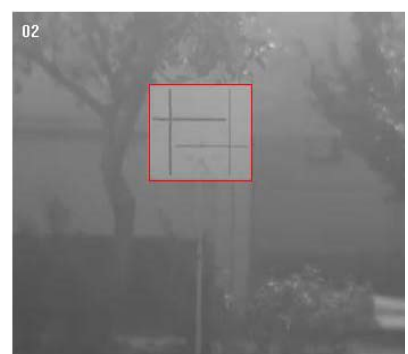

(c) optimal searching step-time NC algorithm(OPT)

Fig.7 Target detection results

Tab.1 The comparison of matching accuracy of the three algorithm

\begin{tabular}{cccccc}
\hline $\begin{array}{c}\text { matching } \\
\text { algorithm }\end{array}$ & $\begin{array}{c}\text { horizontal } \\
\text { step }\end{array}$ & $\begin{array}{c}\text { vertical } \\
\text { step }\end{array}$ & $\begin{array}{c}\text { correlation coefficient of } \\
\text { rough localization }\end{array}$ & $\begin{array}{c}\text { correlation } \\
\text { coefficient of } \\
\text { precise localization }\end{array}$ & $\begin{array}{c}\text { target } \\
\text { detection } \\
\text { position }\end{array}$ \\
\hline TRD & 1 & 1 & 1.000 & 1.000 & $(61,121)$ \\
FIX(1) & 5 & 5 & 0.996 & 1.000 & $(61,121)$ \\
FIX(2) & 20 & 20 & 0.991 & 1.000 & $(61,121)$ \\
FIX(3) & 35 & 35 & 0.965 & 0.968 & $(119,219)$ \\
OPT & 10 & 11 & 0.994 & 1.000 & $(61,121)$ \\
\hline
\end{tabular}

As shown in Tab.1, for fixed step NC algorithm, when step is small, target detection result is 
consistent with the detection results of traditional $\mathrm{NC}$ algorithm. When step is large, correlation coefficient decline drastically, and inaccurate target detection result appear. In addition, target position which is detected by using the optimal searching step-time NC algorithm is consistent with traditional NC algorithm completely, so the matching accuracy is not affected.

\subsection{The comparison of operation time}

The target image is detected by using the traditional $\mathrm{NC}$ algorithm, the fixed step NC algorithm and the optimal searching step-time NC algorithm respectively, and operation time of the three algorithm are recorded. The specific data are as shown in Tab.2.

\begin{tabular}{cccc} 
Tab.2 The comparison of operation time of the three algorithm \\
\hline matching algorithm & horizontal step & vertical step & operation time /s \\
\hline TRD & 1 & 1 & 15.16 \\
FIX(1) & 5 & 5 & 0.43 \\
FIX(2) & 20 & 20 & 0.35 \\
FIX(3) & 35 & 35 & 0.51 \\
OPT & 10 & 11 & 0.22 \\
\hline
\end{tabular}

As shown in Tab.2, the optimal searching step-time NC algorithm has the absolute advantage in operation time, and time of the target image detection is shorten immensely, especially when the size of reference image and template image are larger, the effect is more obvious.

\section{Summary}

According to the real-time performance of target image detection is poor, based on the traditional $\mathrm{NC}$ algorithm, the key factor of matching speed limit is found, and a optimal searching step-time NC algorithm is proposed. Through calculating and setting the optimal searching step-time, the template matching is divided into coarse positioning and fine positioning, and the amount of calculation is greatly reduced, and the real-time performance of template matching is improved. Test results show that the optimal searching step-time $\mathrm{NC}$ algorithm can not only ensure the matching accuracy, but also make matching speed greatly improved when target image is detected, especially for the sequence of target image matching, the effect is more obvious.

\section{References}

[1] Du Dan. Dynamic target detection based on image processing technology[D]. Changchun University of Science and Technology, 2004.

[2] Li Xiaoda. Application of image processing in target detection system[D]. Changchun University of Science and Technology, 2007.

[3] Chen Lifang, Liu yuan, Xu Wenbo. Gray-scale image template matching method that is improved normalization cross-correlation method[J]. Computer Engineering and Applications, 2011, 47(26): 181-183.

[4] Wang Zida, Wang Chunyan. The design of gun stabilization precision measuring device[J]. Journal of Changchun Science and Technology University, 2008, 31(1): 68-70.

[5] Ding L, Goshtasby A, Satter M. Volume image registration by template matching[J]. Image and Vision Computing, 2001, 19: 821-832.

[6] Wang Hongmei, Zhang Ke, Li Yanjun. Research progress on image matching[J]. Computer Engineering and Applications, 2004, 19: 42-44.

[7] Zhang Hongyuan, Chen Zili. Research on classical image matching algorithm and its improved method[J]. Exploitation and Application of Software, 2008, 27(9): 91-94.

[8] Tang Jin, Li Qing. Fast template matching algorithm[J]. Journal of Computer Application, 2010, 30(6): 1559-1561. 